buti, sia quelli dei molti specialisti italiani presenti nel libro, sia quelli di alcuni osservatori britannici attenti come sempre allo sviluppo del nostro sistema politico. Mi piace segnalare, a questo proposito, il capitolo dedicato da John M. Foot alla sinistra di opposizione, che propone tra l'altro la ricostruzione delle vicende di una formazione divenuta oggi cruciale nella vita politica come Rifondazione Comunista, verso la quale invece la scienza politica italiana denuncia una colpevole mancanza di interesse.

Il prodotto finale è dunque un libro che si propone come un reader sul caso italiano denso di stimoli, che fotografa una situazione oggi già «lontana», la prima parte degli anni novanta, proponendo tuttavia una serie di spunti che vale la pena di prendere in considerazione. Tra questi, sicuramente interessante il piccolo saggio di un esperto di cose italiane come lo storico Paul Ginsborg, dedicato alla ricerca dei fattori esplicativi attraverso una ridiscussione efficace, per quanto sintetica, della letteratura recente (altro elemento che stride con molti degli altri capitoli, costruiti invece essenzialmente sulla elaborazione della cronaca giornalistica).

Il libro si chiude con un altro contributo di tutto interesse: una analisi di David Hine sulle prospettive della riforma istituzionale italiana inserite nel contesto dell'evoluzione europea e all'interno di un quadro di riferimento comparativo. I problemi vengono sollevati con puntualità e precisione: in particolare, $\mathrm{i}$ «reali» effetti delle riforme elettorali, la correzione di un sistema parlamentare e il destino di un sistema che rimane coalizionale, a causa della frammentazione partitica. $\mathrm{Ma}$, più che una conclusione, si tratta di una potenziale introduzione ad una ricerca approfondita che avvicini una volta tanto il caso italiano agli altri modelli di democrazia inserendolo correttamente in una ricerca empirica comparata. Una ricerca ancora tutta da perfezionare.

\title{
[Luca Verzichelli]
}

RICHARD J. HERRNSTEIN E CHARLES MURRAY, The Bell Curve: Intelligence and Class Structure in American Life, New York, Free Press, 1994, pp. xxvi-845.

Questo volume, che ha riacceso un aspro e ampio dibattito negli Stati Uniti, tratta di un argomento che gli autori sostengono essere tabù, specie in ambiti accademici e politici: le differenze nei livelli di intelligenza che caratterizzano individui e gruppi e, in particolare, le loro implicazioni sul piano delle politiche pubbliche. L'originalità del libro sta nella centralità accordata a un fattore - le competenze cognitive degli individui e, in particolare, di quelli che beneficiano di politiche sociali - che di solito viene accuratamente evitato nel dibattito po- 
litico per la sua natura altamente controversa. La salienza dell'argomento è testimoniata dal fatto che le reazioni al libro hanno innescato una certa operosità editoriale: The Bell Curve Debate: History, Documents and Opinions, a cura di R. Jacoby e N. Glauberman, Times Books; Measured Lies: The Bell Curve Examined, a cura di J. Kincheloe, S. Steinberg e A. Gresson III, St. Martin's Press; Inequality by Design: Cracking the Bell Curve Myth, di C. Fisher ed altri, Princeton University Press.

Il libro si articola in quattro parti, a loro volta suddivise in ventidue capitoli, e in sette appendici. Nella prima parte del volume gli autori sostengono che sarebbe in atto da decenni una «stratificazione cognitiva» che ha conferito un ruolo dominante ad una «élite cognitiva». Nel corso del ventesimo secolo - grazie all'avvento della scolarizzazione di massa, all'egualitarismo nell'ambito delle opportunità educative e all'efficienza dei meccanismi di selezione - gli individui intellettualmente più dotati sono saliti fino ai vertici della gerarchia occupazionale e hanno reso più efficienti i mercati, eretto barriere che li isolano dagli altri strati della società e, forse, sviluppato interessi politici incompatibili con un regime democratico. Paradossalmente, la democrazia avrebbe avuto così tanto successo nell'eliminazione di alcune divisioni sociali da introdurne una nuova, molto più impermeabile e minacciosa per la democrazia stessa.

La seconda parte è dedicata all'illustrazione dei legami che intercorrono tra i livelli di intelligenza degli individui e l'incidenza di problemi sociali negli Stati Uniti. L'analisi si basa in misura preponderante sulla National Longitudinal Survey of Youth, avviata nel 1979 su un campione di oltre 12.000 giovani, ai quali è stata somministrata anche una batteria di test cognitivi standard. I giovani sono stati assegnati ad una di cinque «classi cognitive» in base alle loro prestazioni sui test (i relativi punteggi si distribuiscono lungo una curva gaussiana, che ha una forma campanulare, donde il titolo del libro). Vengono poi esaminate le relazioni intercorrenti fra questa classificazione e una serie di «comportamenti sociali» - povertà, livelli di istruzione, disoccupazione, gravidanze illegittime e famiglie monogenitoriali, fruizione di sussidi, abuso dei figli, criminalità e comportamento civico (ma non, ad esempio, l'abuso di droghe). Gli autori concludono che, anche controllando per livello di istruzione e per status socio-economico, la propensione a comportarsi in maniera «socialmente indesiderabile» è molto più marcata nelle classi cognitive inferiori.

I capitoli della terza parte (il «contesto nazionale») hanno attirato l'ira dei critici e trattano temi assai delicati, specie nel clima culturale statunitense, intriso di political correctness: le differenze fra gruppi etnici per quanto attiene ai livelli cognitivi e ai comportamenti sociali e l'influenza di tassi di fecondità diversi sulla distribuzione dell'intelligenza. Gli autori illustrano i risultati di molti studi secondo i quali gli americani di origine asiatica hanno un livello medio di intelligenza un 
po' più alto rispetto ai bianchi e i neri ne hanno uno molto inferiore. Essi sostengono, inoltre, che tali differenze non possono essere ricondotte soltanto a distorsioni inerenti alle tecniche di rilevazione delle competenze cognitive, bensì rispecchiano differenze effettive. Non solo, ma queste reali differenze dipenderebbero sì dalle pessime condizioni socio-economiche in cui vivono molti neri, ma anche, e in misura prevalente, da differenze genetiche (da qui nascono le accuse di razzismo lanciate ai due autori). Inoltre, se si tiene conto dei diversi livelli cognitivi dei gruppi etnici, scompaiono le diseguaglianze solitamente osservate sul mercato delle opportunità educative e del lavoro. Ancora, il «capitale cognitivo» della società americana sarebbe soggetto a minacce provenienti dall'elevata fecondità delle donne meno dotate sul piano cognitivo e dalle minori capacità intellettive degli immigrati odierni: il livello cognitivo medio del paese ne soffre, e con esso, in ultima analisi, la qualità della vita di coloro che non appartengono all'élite cognitiva.

È opportuno, prima di procedere, esplicitare (come fanno gli stessi autori) alcuni degli assunti sottesi all'intera opera: l'intelligenza è un costrutto ormai ben consolidato e intorno al quale la comunità scientifica ha formato un robusto consenso; i test di intelligenza riescono a rilevare le competenze cognitive degli individui in maniera affidabile; l'intelligenza è una proprietà determinata in parte da influenze ambientali ma soprattutto dal patrimonio genetico degli individui; di conseguenza, le abilità intellettive di un individuo sono poco malleabili, cioè non possono essere migliorate in misura significativa se non con grande difficoltà. Tutti questi assunti esulano dall'ambito disciplinare proprio degli autori (di cui sono note le inclinazioni politiche di stampo neo-conservatore) e, checché ne dicano loro, sono assai suscettibili di contestazione. Il concetto di intelligenza, e specie la nozione di un'unica intelligenza di carattere generale, è uno dei più dibattuti in campo psicometrico. Le definizioni operative attraverso le quali si giunge a un «quoziente di intelligenza» sono molteplici, e sono tutte afflitte da forme più o meno acute di distorsione, specie quando ci si prefigge di effettuare confronti tra gruppi definiti su base etnica o socio-economica. L'intelligenza di un individuo, per quanto possa avere una componente genetica e quindi immodificabile, dipende comunque anche da fattori ambientali di tipo sanitario, alimentare, familiare ed educativo, per menzionarne soltanto alcuni.

Comunque, abbracciare questi assunti non è necessario per sviluppare molte delle riflessioni più pertinenti degli autori - come peraltro essi stessi riconoscono (per cui non si capisce bene perché li abbiano adottati). Talvolta Herrnstein e Murray non sembrano neppure tanto convinti della fondatezza di questi assunti: in diverse occasioni essi chiamano in causa le difficoltà metodologiche attinenti alla rilevazione dei livelli di intelligenza degli individui. Inoltre, la loro concettualizzazione dell'intelligenza oscilla da un'accezione assai generale, 
corrispondente ad onnicomprensive «qualità personali», a una più specifica che rasenta la razionalità dell'agire (concetto, per il resto, del tutto assente dall'intera trattazione). Una confusione analoga caratterizza la loro nozione di ereditarietà (riferita all'intelligenza), talvolta declinata in termini strettamente biogenetici, talaltra in termini di trasmissione ambientale. È singolare che gli autori abbiano voluto conferire un ruolo così centrale a un concetto così controverso senza dotarlo di confini semantici netti.

L'ultima parte del libro («Vivere insieme») affronta le implicazioni politiche che discendono dal fatto che la maggior parte degli individui cui sono diretti interventi di politica sociale apparterrebbero alle classi cognitive meno dotate; nella misura in cui le politiche pubbliche non tengono conto di questa situazione, esse sarebbero condannate all'insuccesso se non ad esiti controproducenti. In una società avanzata, in cui la scolarizzazione è di massa e le condizioni sanitarie e di alimentazione sono buone, il miglioramento delle condizioni ambientali in cui le competenze cognitive si sviluppano non dà luogo ad alcun effetto apprezzabile.

Herrnstein e Murray prendono di mira, in particolare, le politiche di affirmative action in campo educativo e occupazionale, originariamente introdotte con intenti di tutela verso alcune minoranze etniche: secondo gli autori, esse rafforzano le identità di gruppo anziché promuovere l'ideologia del melting pot, peggiorano di conseguenza i rapporti inter-etnici e di fatto relegano le minoranze «protette» in una cittadinanza di second'ordine. In ultima analisi, la maggior parte delle politiche sociali adottate negli Stati Uniti cercherebbe (inutilmente) di ottenere l'eguaglianza degli esiti anziché garantire l'eguaglianza delle opportunità, e si baserebbe sulla convinzione che ogni disparità sul piano fattuale costituisce necessariamente una diseguaglianza sul piano morale. I rimedi proposti - ridimensionamento del ruolo dell'amministrazione federale, conferimento di maggiori poteri ai governi locali, smantellamento del welfare state, maggiore coinvolgimento delle famiglie, ecc. - si iscrivono direttamente nel filone del pensiero neo-conservatore americano, ma vengono collocati in un contesto molto pessimistico, caratterizzato da un senso di declino inevitabile e, come si è detto, da un crescente isolamento dell'élite, dal peggioramento della qualità della vita degli svantaggiati, dal rafforzamento delle divisioni sociali e forse dal venir meno dei presupposti della democrazia.

Le conclusioni cognitive e operative cui gli autori giungono sono a volte ingiustificatamente perentorie, frutto di una volontà di semplificare e di schierarsi, nonché di una sottovalutazione della difficoltà di fondare scelte fra opzioni politiche su differenze inter-individuali di capacità cognitiva. Per il resto, tuttavia, le tesi, l'amplissima documentazione e i riscontri empirici vengono esposti con equilibrio e competenza. Chi legge a volte si sente a disagio, ma solo perché il volume ha 
il merito di mettere in discussione alcuni elementi ideologici sottesi alla convivenza democratica.

[Giancarlo Gasperoni]

LUCA LANZALACO, Istituzioni organizzazione potere. Introduzione all'analisi istituzionale della politica, Roma, La Nuova Italia Scientifica, 1995, pp. 223.

La letteratura sulle istituzioni è diventata una «industria in crescita». In particolare a partire dalla seconda metà degli anni ottanta, all'interno delle scienze sociali è cresciuta instancabilmente l'attenzione verso le istituzioni. Non poteva essere altrimenti, data l'incapacità dimostrata dal paradigma comportamentista nel rispondere in modo soddisfacente a due quesiti essenziali della ricerca sociale: da che cosa è influenzata sia l'azione individuale che quella collettiva? come e perché si formano e quindi cambiano gli assetti che strutturano le interazioni sociali (ovvero politiche ed economiche)? In entrambi i casi, senza prendere in considerazione la «variabile istituzionale» si fa davvero poca strada. $\mathrm{Ma}$, come è ovvio quando si è in presenza di un'industria in crescita, la presa in considerazione della «variabile istituzionale» è avvenuta secondo approcci scientifici sensibilmente diversi. È opinione condivisa che la dizione di «nuovo istituzionalismo» rinvii, oggi, ad almeno quattro approcci distinti: l'istituzionalismo storico, l'istituzionalismo della scelta razionale, l'istituzionalismo economico e l'istituzionalismo sociologico. Approcci, si badi bene, che si sono sviluppati in relazione ad «oggetti d'indagine» distinti. Se i primi due approcci sono quelli predominanti all'interno della scienza politica, il terzo è particolarmente influente negli studi di economia e di storia economi$\mathrm{ca}$, mentre l'ultimo costituisce l'approccio più significativo all'interno degli studi di sociologia dell'organizzazione. Pur non concettualizzando questa distinzione di approcci scientifici, il libro di Lanzalaco vuole essere un tentativo di costruire un paradigma unitario attraverso la ricomposizione del quarto e del primo approccio, pur con il supporto di alcune categorie proprie del terzo. E, di converso, la sua bête noir è l'istituzionalismo razionalista, ritenuto (in maniera, forse, eccessivamente drastica) incapace di superare la logica economica da cui esso deriva. Infatti, sulla base del proprio background di studi nel campo della teoria della organizzazione, Lanzalaco propone una teoria delle istituzioni che concettualizza queste ultime come «manufatti culturali», oltre che come «strutture regolative». Conseguentemente, la sua definizione di istituzioni include, al loro interno, i sistemi simbolici, gli schemi cognitivi e i codici normativi: e non solo le regole, le procedure e le norme di cui si sono finora preoccupati gli scienziati della politica di impianto istituzionalista. In questo modo, l'interpretazione 